\title{
Ultra-short wavelength operation of a thulium doped fiber laser in the 1620-1660nm wavelength band
}

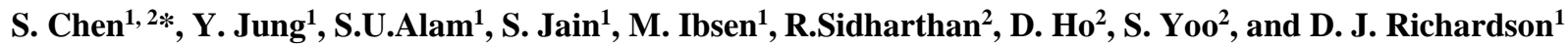 \\ (1) Optoelectronics Research Centre, University of Southampton, SO171BJ, United Kingdom \\ (2) The Photonics Institute, School of Electrical and Electronic Engineering, Nanyang Technological University, Singapore 639798 \\ Sc4f15@soton.ac.uk
}

Abstract: We present a tunable thulium-doped fiber laser (TDFL) incorporating a Tm/Ge co-doped

fiber capable of accessing the U-band wavelength region (1620-1660nm). These results represents

by far the shortest laser wavelengths so far for a TDFL.

OCIS codes: (140.3600) Lasers, tunable; (140.3510) Lasers, fiber; (060.3735) Fiber Bragg gratings

\section{Introduction}

Thulium-doped silica fiber has recently emerged as a very attractive gain medium for optical amplification in the $2 \mu \mathrm{m}$ transmission window [1], as well as for power scalable laser sources operating in this 'eye-safe' wavelength range. Broadband operation of tunable thulium doped fiber lasers (TDFL) has been extensively investigated over the past few years and efficient laser emission can readily be achieved in the $1700 \mathrm{~nm}$ to $2050 \mathrm{~nm}$ wavelength range [2-3]. However, it is challenging to achieve shorter wavelength operation of Tm-doped silica fiber, due to the fact that Tm exhibits a strong three-level behavior and as such requires a high population inversion (thus high pump power) and a low cavity loss to achieve reasonable lasing at this waveband. Recently, a tunable TDFL operable over the 1660 $1750 \mathrm{~nm}$ has been demonstrated using a highly wavelength selective fiber Bragg grating (FBG) and a low loss all fiber laser cavity [4]. In addition, by suppressing the amplified spontaneous emission (ASE) at the longer wavelength region $(1800-2000 \mathrm{~nm})$, Li et al have demonstrated a thulium-doped fiber amplifier (TDFA) working in the 1650-1700 nm range with a small signal gain of up to $29 \mathrm{~dB}$ [5]. Operation of Tm-doped fiber devices at or below 1650nm however remains a topic of great interest, not least as it could potentially provide for seamless silica based rare-earth doped amplifier solutions extending from the S-band (exploiting erbium doping) right up to the IR absorption edge of silica fiber.

Very recently, we have explored a new glass composition (specifically $\mathrm{Tm} / \mathrm{Ge}$ co-doped silica fiber) and have successfully demonstrated a wideband tunable TDFL operable from 1679nm to $1992 \mathrm{~nm}$ [6]. In this follow-on work, we focus on ultra-short wavelength operation of the TDFL and present the first demonstration of a tunable TDFL (based on an in-house fabricated Tm/Ge co-doped silica fiber) operating over the 1620-1660nm wavelength band, i.e. extending operation down to the long wavelength edge of the L-band. Our results represent by far the shortest laser wavelengths ever achieved for a TDFL.

\section{Experimental setup}

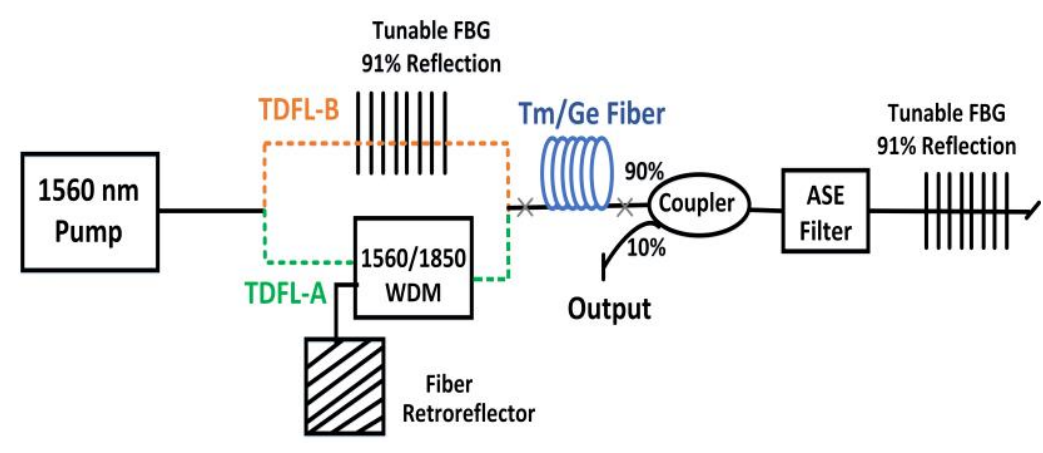

(a)

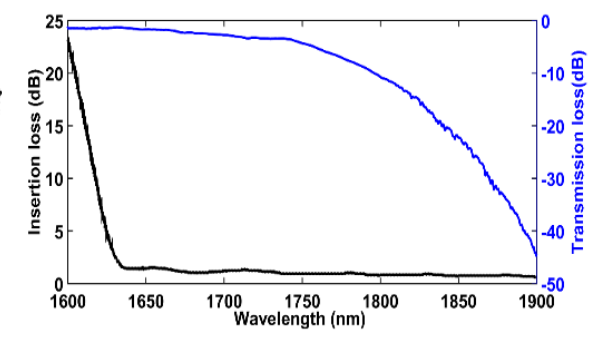

(b)

Fig. 1 (a) Schematic of the TDF, showing the two laser configurations studied, hereafter referred to as TDFL-A (Green) and TDFL-B (Brown), and (b) the insertion loss of the WDM coupler (black) and transmission loss of the ASE filter (blue).

Fig. 1 (a) shows the experimental setup of the all-fiber tunable TDFL illustrating the two laser configurations studied. The setup comprises an in-house built $\mathrm{Er} / \mathrm{Yb}$ fiber laser at $1565 \mathrm{~nm}$ with maxmium output power of $5 \mathrm{~W}$ as the pump source and a $\sim 4.5 \mathrm{~m}$ length of in-house fabricated $\mathrm{Tm} / \mathrm{Ge}$ co-doped fiber with high Ge concentration (15 mol\%). The core diameter and NA of the fiber was measured to be $4.4 \mu \mathrm{m}$ and $\sim 0.28$. In TDFL-A, the pump light is coupled 
into the $\mathrm{Tm} / \mathrm{Ge}$ co-doped fiber through a broadband, filter-based, 1560/1850 nm wavelength division multiplexer (WDM). In order to build the cavity, the signal port of the WDM was spliced to a silver coated fiber refro-reflector, whereas a highly wavlength selective compressible fiber Bragg grating (FBG) with a central wavelength of $\sim 1675 \mathrm{~nm}$ was spliced to the other end of the gain medium. The FBG could be tuned from 1620nm through to $1660 \mathrm{~nm}$ by compression tuning [7]. The measured reflectivity and bandwidth of the FBG varied from $91 \%$ to $94 \%$ and from $0.67 \mathrm{~nm}$ to $1 \mathrm{~nm}$ respectively, depending on the operating wavelength. The 10\% port of the 90/10 tap coupler was used to extract the output from the TDFL. A coiled length of dispersion compensating fiber (DCF) was used as an ASE filter. The bend diameter was optimised to provide low transmission loss at wavelengths below $1700 \mathrm{~nm}$, whilst exhibiting high transmission loss at longer wavelengths. Fig 1(b) shows the bend dependent loss of the coiled DCF for an optimised bend diameter of $5 \mathrm{~cm}$. The insertion loss of the WDM was also measured (see Fig. 1(b)), and it was found that the loss increases significantly at wavelengths shorter than $1640 \mathrm{~nm}$. Therefore, for improved performance at wavelegnths below $1660 \mathrm{~nm}$ the TDFL-B configuration was used. Here the WDM and retro-reflector were replaced with a second compressible FBG thereby avoiding the insertion loss of the WDM. Given that the FBG is wavelength selective, it was possible to couple $1565 \mathrm{~nm}$ pump power through the FBG without introducing additional cavity loss. Tuning of the laser cavity was realized by simultaneously compressing the two FBGs (mounted on mechanical stages) whilst ensuring wavelength sychronization between the two. Splicing the Tm/Ge co-doped fiber and the SMF-28 fiber pigtails of the passive components togther resulted in a high splice loss of $\sim 3 \mathrm{~dB}$ due to the large mode field diameter mismatch and this compromised the short wavelength operation of the cavity. To reduce this splice loss, a short section $(\sim 1 \mathrm{~cm})$ of OFS TmDF200 was used as an intermediate fiber between SMF-28 and Tm/Ge co-doped fiber and the optimized splice loss was reduced down to $0.8 d B$. The $90 / 10$ output coupler has an insertion loss of around $0.4 d B$ in the 1550-1750 nm waveband. The output characteristics of the tunable laser was measured by using a Yokogawa optical spectrum analyser (AQ6375) and a thermal power meter (Ophir 3A-FS).

\section{Results and discussion}

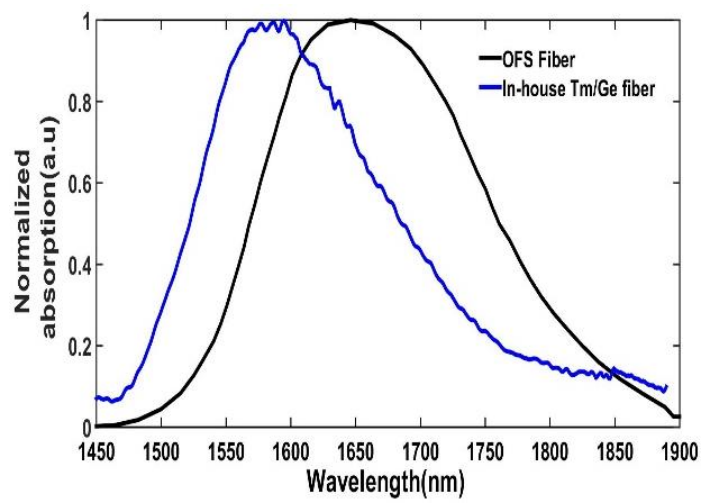

Fig. 2 Normalized absorption curves for OFS Tm-doped fiber (black) and in-house fabricated Tm/Ge co-doped fiber (blue).

As shown in Fig. 2, the in-house fabricated Tm/Ge co-doped fiber exhibits a blue-shifted absorption curve with a peak absorption of $\sim 20 \mathrm{~dB} / \mathrm{m}$ at $1580 \mathrm{~nm}$, significantly shorther than the commerical Tm-doped aluminosilicate fiber. Moreover, according to McCumber theory, the emission profile of $\mathrm{Tm} / \mathrm{Ge}$ co-doped fiber will also be blue-shifted, enabling shorter wavelength operation. In the TDFL-A configuration, the high insertion loss of the WDM coupler and ASE filter as well as the splice losses between SMF-28 and Tm/Ge co-doped fibers give an aggregate single pass cavity loss of $6 d B$ at $1650 \mathrm{~nm}$, which increased further at shorter wavelengths. To achieve short wavelength operation, a short $\mathrm{Tm} / \mathrm{Ge}$ co-doped fiber length is preferred in order to avoid signal reabsorption, with the minimum usable fiber length determined by the requirement to achieve sufficient total gain to overcome the large cavity loss. In our experiment, we found that the optimal fiber length was $4.5 \mathrm{~m}$ (for a pump power of $3.5 \mathrm{~W}$ ). The blue colored plot in Fig. 3(a) shows the output power as a function of operating wavelength for the maximum available pump power. A highest output power of $\sim 15 \mathrm{~mW}$ was obtained at a wavelength of $1660 \mathrm{~nm}$. The shortest lasing wavelength was measured to be $1637 \mathrm{~nm}$ with an output power of $\sim 5 \mathrm{~mW}$. The red colored plot in Fig. 3(a) shows the output power as a function of operating wavelength for the TDFL-B laser configuration. The same pump power and fiber length were used to ensure a fair comparison between the two laser configurations. We found that the lasing threshold for 1660nm operation decreased from $1.6 \mathrm{~W}$ to $0.9 \mathrm{~W}$, resulting in a much higher laser output power. Also, an almost constant output power was achieved over the entire tuning range from $1620 \mathrm{~nm}$ to $1660 \mathrm{~nm}$ which highlights the importance of avoiding passive components whose insertion loss varies with wavelength (i.e. the WDM coupler). The highest output 
power of about $22 \mathrm{dBm}$ was recorded at $1660 \mathrm{~nm}$ with a slope of efficiency of $5.8 \%$ with respect to the launched pump power, with an output power of $17 \mathrm{dBm}$ measured at $1620 \mathrm{~nm}$, the shortest wavelength we could reach with the current tunable gratings.

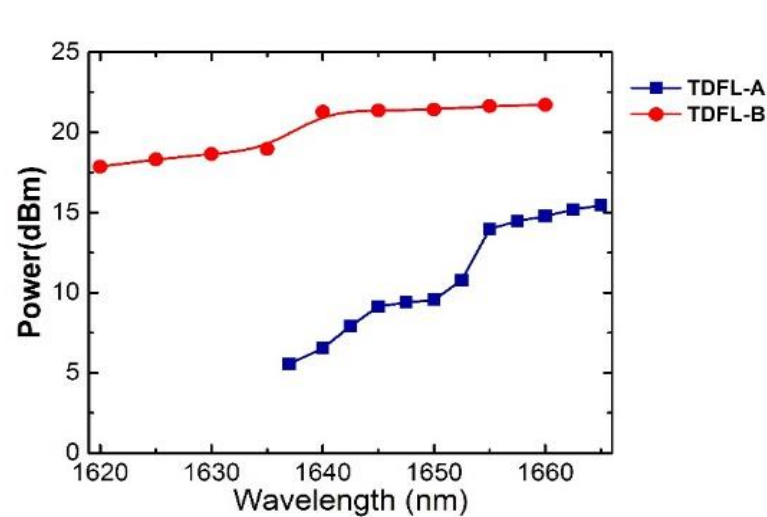

(a)

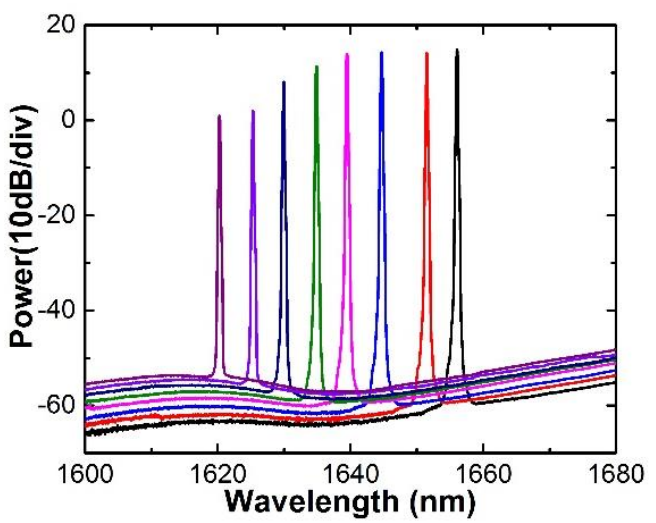

(b)

Fig. 3 (a) Output powers of TDFL-A and TDFL-B for different lasing wavelengths, and (b) Output optical spectra of the TDFL-B laser configuration (measured with $0.1 \mathrm{~nm}$ OSA resolution).

Since the output from the laser cavities was extracted after reflection from FBG1, the out-of-band ASE was well suppressed, resulting in a high optical signal-to-noise ratio (OSNR) albeit at a slightly lower output power. Figure $3 \mathrm{~b}$ plots the spectral characteristics of TDFL-B. An OSNR of more than $45 \mathrm{~dB}$ was successfully achieved across the full tuning range from $1620 \mathrm{~nm}$ to $1660 \mathrm{~nm}$ (up to $60 \mathrm{~dB}$ OSNR from $1640 \mathrm{~nm}$ to $1660 \mathrm{~nm}$ ). The $3 \mathrm{~dB}$ bandwidth of the laser lines were measured to be $<0.3 \mathrm{~nm}$. We believe that the output power and lasing efficiency could be further improved by optimizing the ASE filter to provide a much sharper wavelength cutoff and the $\mathrm{Tm} / \mathrm{Ge}$ fiber glass composition.

\section{Conclusion}

We have successfully demonstrated an all-fiber tunable TDFL operating over the $1620 \mathrm{~nm}$ to $1660 \mathrm{~nm}$ range (communication U-band) by using $\mathrm{Tm} / \mathrm{Ge}$ co-doped fiber. This unique glass composition effectively shifts the emission cross-section of the Tm ions to shorter wavelengths as compared to conventional Tm-doped fiber using an aluminosilicate host. This greatly improved short wavelength operation and allowed us to bridge the gap between $\mathrm{Er}^{3+}$ and $\mathrm{Tm}^{3+}$ ions in a silica based glass host. Using two FBGs as cavity mirrors and a long wavelength ASE filter, more than $17 \mathrm{dBm}$ output power with $>45 \mathrm{~dB}$ OSNR was achieved over the entire tuning range.

\section{Acknowledgement}

This work was supported by Academic Research Fund Tier 1, Ministry of Education (Singapore) and by the EPSRC funded Hollow Core Fiber Photonics Programme Grant (EP/P030181/1).

\section{References}

[1] Z. Liu, Becker et al., "High-Capacity Directly Modulated Optical Transmitter for 2- $\mu$ m Spectral Region” J. Lightwave Technol. 33, 1373 (2015).

[2] W. A. Clarkson et al., "High-power cladding-pumped Tm doped silica fiber laser with wavelength tuning from 1860 to $2090 \mathrm{~nm}$," Opt. Lett. 27(22), 1989-1991 (2002).

[3] C. Guo et al., "High-power and widely tunable Tm-doped fiber laser at $2 \mu \mathrm{m}$," Chin. Opt. Lett. 10(9), 091406 (2012).

[4] J. M. O. Daniel et al., "Ultra-short wavelength operation of a thulium fibre laser in the 1660-1750 nm wavelength band," Opt. Express 23, $18269-18276(2015)$

[5] Z. Li et al., "Exploiting the short wavelength gain of silica-based thulium-doped fiber amplifiers," Opt. Lett. 41, 2197-2200 (2016).

[6] Chen et al., "Ultra-wideband Operation of a Tunable Thulium Fiber Laser offering Tunability from1679-1992 nm", in ECOC (2017), M.2.B.4.

[7] Mokhtar et al., "Fiber Bragg grating compression-tuned over 110 nm", Electronics Letters, Vol. 39, No. 6, 2003 